\title{
The Effect of Additional Embryo Transfer on the Pregnancy Rate in Young Women Receiving in vitro Fertilization: A Natural Experiment Study
}

This article was published in the following Dove Press journal: International Journal of Women's Health

\author{
Ran Kim ${ }^{\prime}$ \\ Seung-Ah Choe $\mathbb{D D}^{2}$ \\ Eun A Park' \\ Myung Joo Kim' \\ Young-Sang Kim (1D \\ You Shin Kim' \\ 'Department of Obstetrics and \\ Gynecology, CHA Fertility Center Seoul \\ Station, CHA University School of \\ Medicine, Seoul, 04637, Korea; \\ ${ }^{2}$ Department of Preventive Medicine, \\ Korea University College of Medicine, \\ Seoul, 0284I, Korea
}

Purpose: To assess the impact of additional embryo transfer (ET) on pregnancy in young women, we used a natural-experiment approach.

Patients and Methods: The design was based on the national policy of South Korea limiting the number of embryos transferred in vitro fertilization (IVF): $\leqq 2$ embryos on day 2-4 or one on day 5-6 for patients aged $<35$, with one extra embryo allowed for patients aged $\geq 35$. Using the data from 1909 ET cycles of 1287 women aged $\geq 34$ and $\leq 35$, we calculated adjusted risk ratios (RRs) for pregnancy.

Results: Half of cycles were undertaken by women aged 35, and additional ET was performed in $68.7 \%$ of them. Intrauterine pregnancy $(45.2 \%$ vs $51.3 \%)$ and multiple gestation $(30.5 \%$ vs $6.9 \%)$ were more common in women aged 35 than in those aged 34 . The RR for intrauterine pregnancy was 1.34 (95\% confidence interval: 1.12-1.59) when comparing double ET to single ET in frozen day 5-6 cycles.

Conclusion: We observed no evidence of a higher probability of pregnancy with additional ET in fresh or frozen day 3-4 ET, or in fresh day 5-6 ET of women aged 35. Additional ET may not increase the successful pregnancy rate in the 35 -year-old group, unless it is a frozen day 5-6 ET cycle.

Keywords: in vitro fertilization, single embryo transfer, pregnancy rate, multiple pregnancy

\section{Introduction}

In the process of in vitro fertilization, many medical practitioners are transferring two or more embryos for better success rate of IVF, though single embryo transfer (ET) is recognized as the standard of care for good-prognosis patients. ${ }^{1-3}$ Countries therefore legislate the number of embryos that may be transferred during $\mathrm{IVF}^{4}$ because limiting the number of embryos transferred can minimize the risk of multiple gestation without affecting pregnancy rate. ${ }^{5,6}$ Generally, the recommendation on the number of embryos to transfer depends on the patient's age: one or two for women aged 35-36 years, and as many as three for those of a more advanced age. ${ }^{7,8}$

Simple observational studies on the impact of different treatments may be inaccurate due to confounding factors and reverse causality. ${ }^{9}$ Natural experiments are defined as a research approach which evaluates large-scale population health interventions that are not amenable to experimental allocation. ${ }^{9}$ This approach enables researchers to measure the changes in outcome that may be plausibly attributed to the intervention. ${ }^{10-12}$

In 2015, the Ministry of Health and Welfare (MoHW) of South Korea published a policy prescribing the number of embryos to transfer, discouraging medical
Correspondence: Seung-Ah Choe Department of Preventive Medicine, Korea University College of Medicine Department of Epidemiology \& Health Informatics, Graduate School of Public Health, Korea University, 73 Goryeodaero, Seongbuk-gu, Seoul, 0284I, Korea Tel +82-2-2286- I 344

Email seungah@korea.ac.kr
International Journal of Women's Health 2021:13 379-384 
professionals from transferring more than three (for 3-4day ET) or two (for 5-6-day ET) embryos, regardless of embryo grade, to reduce multiple gestations. ${ }^{13}$ For women younger than 35 years, no more than two day 3-4 embryos or one day 5-6 embryo should be transferred. ${ }^{13,14}$ The MoHW regularly evaluates all IVF centers to verify whether they are following the national policy. According to this policy, a woman aged 34 who fails to conceive after the transfer of two cleavagestage embryos or one blastocyst becomes eligible for the transfer of three cleavage-stage embryos or two blastocysts soon after their 35th birthday, regardless of the number of good embryos (Figure 1). This enabled us to perform a natural experiment among 34-35-year-old women, as a woman's general condition is assumed to be not changed radically during this transition in age. Our objective was to assess the impact of national ET guideline of South Korea allowing additional ET after a patient's 35th birthday on the success rate of intrauterine pregnancy, by analyzing the data of women aged $\geq 34$ and $\leq 35$ who have undertaken fresh or frozen ET cycles using natural experiment approach.

\section{Patients and Methods}

\section{Study Population}

This was a retrospective study of patients (aged $\geq 34$ and $\leq 35$ ) who had undergone ET cycles in 2015-2018 at a single IVF center in Seoul, South Korea. We identified 2785 fresh or frozen ET cycles conducted in this population. We excluded cycles following a freeze-all protocol, cycles cancelled due to failed oocyte retrieval, and cycles where no transferrable embryo was obtained; accordingly, we restricted our analysis to 1909 fresh or frozen IVF-ET cycles undertaken by 1287 women. As a retrospective chart review study, this research met the criteria for waiver of written informed consent and approved by the institutional review board of Gangnam CHA Hospital (approval number: GCI-18-48). This study was conducted ensuring patient data confidentiality and in accordance with the Declaration of Helsinki.

\section{IVF-ET Procedure}

All patients had undergone controlled ovarian stimulation, as described previously. ${ }^{15}$ In brief, most of the participants received a gonadotropin-releasing hormone (GnRH)antagonist protocol, with the initiation of recombinant follicle-stimulating hormone (r-FSH; Gonal- $\mathrm{f}^{\mathbb{R}}$; Merck Serono, Darmstadt, Germany or Follitrope ${ }^{\circledR}$; LG Life Sciences Ltd., Seoul, South Korea) therapy from menstrual day 3, after baseline transvaginal ultrasonography (TVS) to ensure ovarian quiescence. Daily r-FSH dose ranged from 150 to $225 \mathrm{IU}$. Follicular monitoring was performed from day 7 to day 8 with TVS, and the dose was adjusted according to follicular development. When a dominant follicle of $\geq 15 \mathrm{~mm}$ in diameter was detected, GnRH-antagonist (ganirelix acetate, Ganilever ${ }^{\circledR}$; LG Life Sciences Ltd.) treatment was started at a dose of $0.25 \mathrm{mg} /$ day subcutaneously, until the day of ovulation. Recombinant human chorionic gonadotropin (hCG; Ovidrel $^{\circledR}$; Merck Serono) was administered when there was at least one follicle of $\geq 18 \mathrm{~mm}$ in diameter. After TVS-guided oocyte retrieval and insemination, embryos were cultured and assessed. Following the national

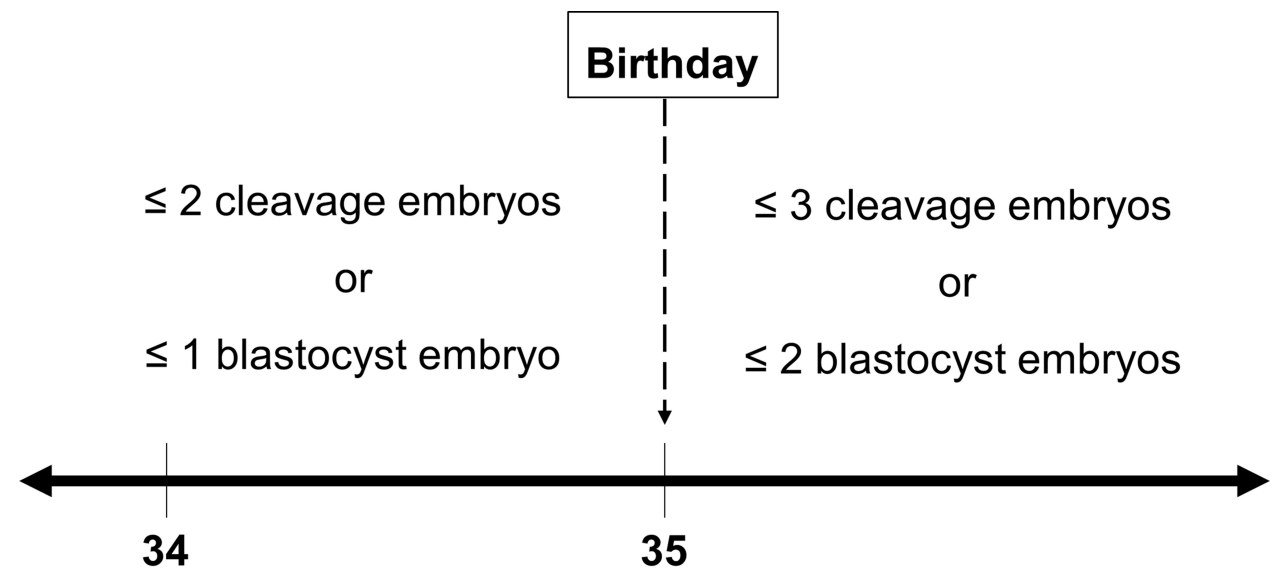

\section{Women's age}

Figure I Eligibility for additional embryo transfer in women aged 34 and 35 according to the policy of the Ministry of Health and Welfare of Korea. Abbreviation: ET, embryo transfer. 
guideline, we transferred no more than two cleavage-stage embryos or one blastocyst in 34-year-old patients, and no more than three cleavage-stage embryos or two 5-6 blastocysts in 35-year-old patients. Luteal support was provided for all patients with daily vaginal progesterone (P4) suppositories or intramuscular P4 injection from the day of oocyte retrieval. For patients undertaking a frozen ET cycle, we started administering a daily $6 \mathrm{mg}$ of oral estradiol valerate (Progynova ${ }^{\circledR}$; Schering [Korea] Ltd., Seoul, South Korea) from menstrual day 3. When endometrial thickness reached approximately $8 \mathrm{~mm}$, luteal support was provided in the form of daily vaginal or intramuscular P4. Thawed embryos were transferred 2-6 days later, depending on the day of embryo freezing.

\section{Outcome and Covariates}

The main outcome measures were intrauterine pregnancy and multiple gestations, determined via serum hCG measured in gestational week 4, and TVS performed in week 5-6. Biochemical or ectopic pregnancy was defined as serum $\mathrm{hCG}>20 \mathrm{IU} / \mathrm{mL}$ in week 4 and the absence of an intrauterine gestational sac in week 5-6. We defined intrauterine pregnancy as the presence of one or more gestational sacs in week 5-6.

We also assessed baseline clinical characteristics for fresh ET cycles, such as thin endometrium $(<7 \mathrm{~mm})$, elevated serum P4 $(>1.5 \mathrm{ng} / \mathrm{mL})$ at the time of hCG injection, decreased ovarian reserve (total number of oocytes $<5$ ), and season of embryo transfer. For frozen ET cycles, thin endometrium and season of embryo transfer were assessed as baseline clinical characteristics.

\section{Statistical Analysis}

We first compared baseline clinical characteristics between the ET cycles conducted in the 34- and 35-year-old groups. This included mean endometrial thickness on the day of hCG injection; number of oocytes, fertilized eggs, and transferred embryos; P4 concentration on the day of hCG initiation; the proportions of fresh ET and blastocyst transfer; and the proportion of intrauterine pregnancies, biochemical or ectopic pregnancies, and multiple gestations.

Continuous variables were compared between two groups using Student's $t$-test. Pearson's Chi-squared test with Yates' continuity correction was used to compare categorized variables. Further, we calculated unadjusted and adjusted risk ratios (RRs) of intrauterine pregnancy and multiple gestations for additional ET, including baseline characteristics as covariates to adjust for potential confounding effects. As some patients were included more than once, we performed logistic regression using a generalized linear mixed models to account for intraindividual differences in IVF outcomes. ${ }^{15}$ All analyses were performed using the open source software package, $\mathrm{R}$ ver. 3.6.2 (R Core Team, 2019, Vienna, Austria).

\section{Results}

Of the 1909 ET cycles, half of the cycles (51.2\%) were conducted in patients aged 35. In all ET cycles performed in this age group, additional ET was performed in $68.7 \%$ of patients. There were no differences in endometrial thickness, serum P4 level on the day of hCG initiation, or number of retrieved oocytes or fertilized eggs (Table 1). More embryos were transferred in the 35-year-old group than in the 34-yearold group. The overall intrauterine pregnancy rate was $6.1 \%$ higher in 35-year-old women than in 34-year-old women (51.3\% vs $45.2 \%, \mathrm{P}<0.001)$. Biochemical or ectopic pregnancies occurred in $4.5 \%$ of cycles in both groups. In cycles yielding intrauterine pregnancy, the 35-year-olds experienced a higher $(\mathrm{P}<0.001)$ multiple gestation rate $(30.5 \%)$ than did the 34-year-olds $(6.9 \%)$.

Upon comparing stratified proportions, the intrauterine pregnancy rate with fresh cycles was similar between patients receiving additional ET cycles and those who did not, both for day 2-4 and day 5-6 transfers (Table 2). In patients receiving frozen ET cycles of day 5-6 embryos, the intrauterine pregnancy rate was higher $(\mathrm{P}<0.001)$ in patients receiving additional $\mathrm{ET}$ than in those not receiving additional ET $(71.7 \%$ vs $52.9 \%)$. The multiple pregnancy rate was higher in patients who had received day 5-6 ET than in patients who had received day 2-4 ET, both for fresh (36.0\% vs $0.0 \%$ ) and frozen $(44.0 \%$ vs $0.0 \%)$ ET cycles.

According to our generalized linear mixed models, the adjusted RR of intrauterine pregnancy upon additional ET was 1.16 (95\% confidence interval [CI]: 0.84-1.60) for fresh day 2-4 cycles and 0.93 (95\% CI: 0.66-1.30) for fresh day 5-6 cycles, with no difference for any of these (Figure 2). In patients undergoing frozen day 2-4 ET cycles, the RR of intrauterine pregnancy upon additional ET was 0.67 (95\% CI: 0.25-1.79). In patients undergoing frozen day 5-6 ET cycles, additional ET increased the probability of intrauterine pregnancy $(1.34,95 \% \mathrm{CI}: 1.12-1.59)$.

\section{Discussion}

We used a natural-experiment approach to assess the impact of national ET guideline of South Korea allowing additional ET after a patient's 35th birthday on pregnancy 
Table I Characteristics of 1909 Fresh and Frozen Embryo Transfer Cycles of 34-35-Year-Old Women

\begin{tabular}{|l|c|c|c|}
\hline Variables & 34 Years (932 Cycles) & 35 Years (977 Cycles) & P for Difference \\
\hline Endometrial thickness on hCG day (mm) & $9.56 \pm 2.1$ & $9.63 \pm 2.18$ & 0.839 \\
P4 on hCG day (ng/mL) & $0.83 \pm 0.57$ & $0.86 \pm 1.00$ & 0.587 \\
Number of oocytes & $12.79 \pm 9.04$ & $13.3 \pm 10.16$ & 1.000 \\
Number of fertilized eggs & $8.24 \pm 6$ & $8.75 \pm 6.89$ & 0.799 \\
Number of transferred embryos, day 2-4 & $1.85 \pm 0.43$ & $2.47 \pm 0.69$ & $<0.00 \mathrm{I}$ \\
Number of transferred embryos, day 5-6 & $1.04 \pm 0.19$ & $1.75 \pm 0.44$ & $<0.00 \mathrm{I}$ \\
Proportion of fresh embryo transfer & $60.1 \%(818 / 1360)$ & $63.2 \%(900 / 1425)$ & 1.000 \\
Proportion of blastocyst transfer & $42.9 \%(400 / 932)$ & $46.9 \%(458 / 977)$ & 1.000 \\
Thin endometrium (<7mm) on hCG day & $5.8 \%(52 / 895)$ & $6.0 \%(56 / 940)$ & 0.972 \\
Serum progesterone elevation on hCG day & $13.5 \%(109 / 807)$ & $13.1 \%(117 / 893)$ & 0.320 \\
Decreased ovarian reserve (retrieved oocytes <5) & $21.4 \%(173 / 809)$ & $17.1 \%(153 / 896)$ & 0.064 \\
Intrauterine pregnancy & $45.2 \%(421 / 932)$ & $51.3 \%(501 / 977)$ & 0.009 \\
Biochemical or ectopic pregnancy & $4.5 \%(20 / 44 \mathrm{I})$ & $4.5 \%(20 / 445)$ & 0.198 \\
Multiple pregnancy of all intrauterine pregnancy & $6.9 \%(29 / 42 \mathrm{I})$ & $30.5 \%(153 / 501)$ & $<0.00 \mathrm{I}$ \\
\hline
\end{tabular}

Notes: Continuous variables are presented as means and standard errors and binary variables are as proportions (and frequencies in parentheses). Difference in continuous variables was tested using Student $t$-tests. Pearson's Chi-squared test with Yates' continuity correction was used for categorized variables.

Abbreviations: hCG, human chorionic gonadotropin; P4, progesterone.

Table 2 Frequencies of Intrauterine Pregnancy and Multiple Pregnancy Among Intrauterine Pregnancy Cases, 1909 Cycles of 34-35Year-Old Women

\begin{tabular}{|c|c|c|c|c|}
\hline & ET Day & No Additional ET & Additional ET & $P$ for Difference \\
\hline \multicolumn{5}{|c|}{ Intrauterine pregnancy } \\
\hline \multirow[t]{2}{*}{ Fresh cycles } & Day 2-4 & $32.6 \%(128 / 393)$ & $36.0 \%(62 / 172)$ & 0.479 \\
\hline & Day 5-6 & $51.5 \%(67 / 130)$ & $46.0 \%(75 / 163)$ & 0.411 \\
\hline \multirow[t]{2}{*}{ Frozen cycles } & Day 2-4 & $28.2 \%(20 / 7 I)$ & $17.2 \%(5 / 29)$ & 0.373 \\
\hline & Day 5-6 & $52.9 \%(324 / 613)$ & $71.7 \%(24 I / 336)$ & $<0.001$ \\
\hline \multicolumn{5}{|c|}{ Multiple pregnancy } \\
\hline \multirow[t]{2}{*}{ Fresh cycles } & Day 2-4 & $18.8 \%(24 / \mid 28)$ & $27.4 \%(17 / 62)$ & 0.191 \\
\hline & Day 5-6 & $0.0 \%(0 / 67)$ & $36.0 \%(27 / 75)$ & $<0.001$ \\
\hline \multirow[t]{2}{*}{ Frozen cycles } & Day 2-4 & $30.0 \%(6 / 20)$ & $0.0 \%(0 / 5)$ & 0.289 \\
\hline & Day 5-6 & $0.0 \%(0 / 324)$ & $44.0 \%(106 / 24 I)$ & $<0.001$ \\
\hline
\end{tabular}

Notes: Additional ET cycle is defined as transfer of three day 2-4 embryos or two day 5-6 embryos which is allowed after 35th birthday. Proportions were compared using Fisher's exact test or chi-square test. Multiple pregnancy rate was calculated only in intrauterine pregnancy cases. Two ET cycles were missing for ET day. Sum of all numerators and denominators may not equal to the total number of ET cycles due to cases with missing information.

Abbreviation: ET, embryo transfer.

rate, using IVF-ET data of women aged $\geq 34$ and $\leq 35$. Major finding is that the overall pregnancy rate in women aged 35 was higher than those aged 34 in our study population. There was a positive association between additional ET and an increased intrauterine pregnancy rate was evident only for frozen day 5-6 ET cycles. For fresh and frozen day 2-6 ET cycles and frozen day 2-4 ET cycles, there was no apparent association between additional ET and the intrauterine pregnancy rate. This finding suggests that, for women aged 35 who decide to undergo fresh blastocyst or any types of cleavage ET, additional ET may not yield an increased probability of pregnancy. This finding would add an evidence to assist practitioners and patients in weighing the benefits of additional ET against the risk of multiple gestations in this age group.

Prior studies revealed pregnancy rates in single ET are comparable to or slightly lower than those in additional ET 


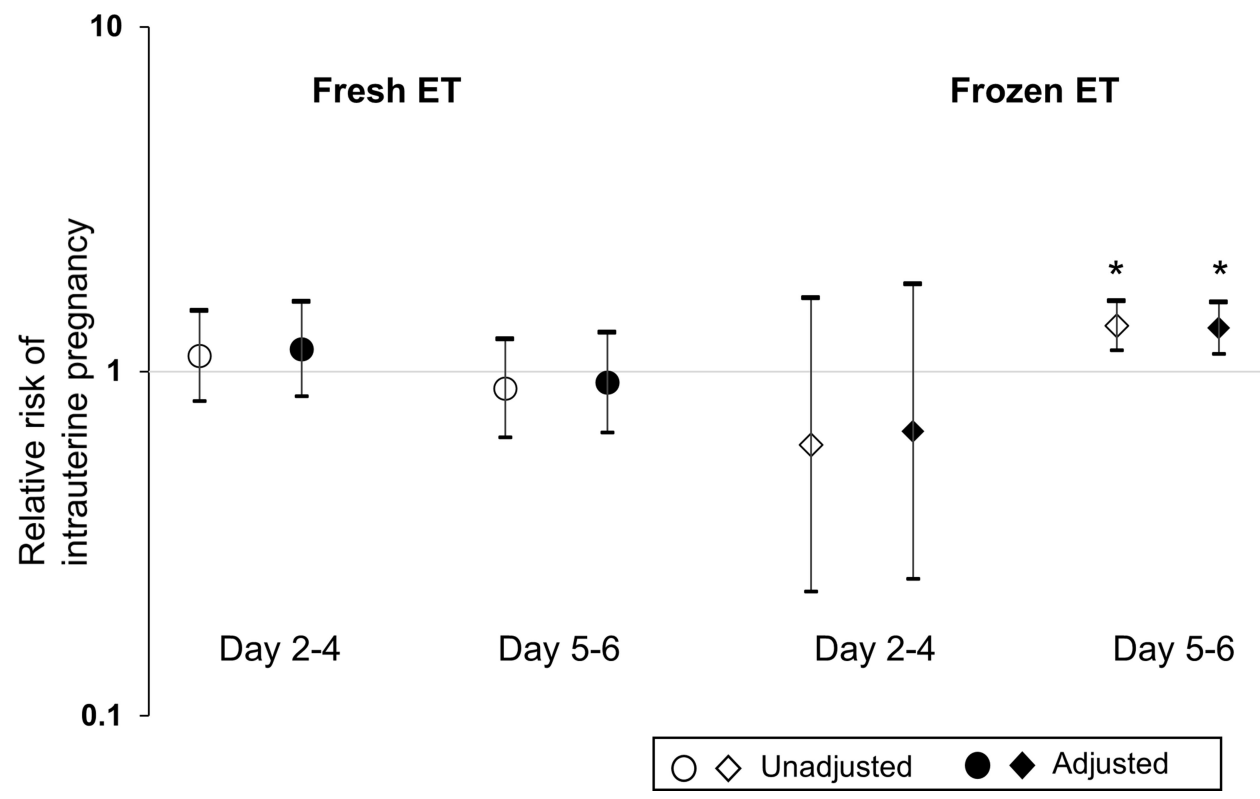

Figure 2 Unadjusted and adjusted relative risk of intrauterine pregnancy for additional embryo transfer, 1909 cycles of 34-35-year-old women.

Notes: In the models for fresh cycles, thin endometrium, P4 elevation, decreased ovarian reserve, season of transfer and within-individual correlation were included as covariates. In models for frozen cycles, thin endometrium, season of transfer and within-individual correlation were included. Hollow markers represent unadjusted and solid markers represent adjusted estimates. Asterisks $(*)$ indicate $p<0.05$

Abbreviation: ET, embryo transfer.

with a significant reduction of multiple pregnancy risk. According to one systematic review, the live-birth rate following repeated single ET was not different from that following a single cycle of double ET; this suggests that repeated single ET is the best option for most women undergoing IVF-ET, considering the comparable cumulative pregnancy rate and the odds of multiple gestation. ${ }^{6}$ However, the financial, physical, and emotional cost of additional cycles, and worse prognosis in women of advanced child-bearing age remain a concern. ${ }^{1}$ A metaanalysis using pooled IVF data revealed that double ET is associated with a higher ongoing pregnancy and live birth rate than single $\mathrm{ET},{ }^{16}$ while other authors have argued that repeated single ET yields comparable pregnancy rates to that of double ET. ${ }^{17}$ We postulate that the effect of additional ET reported in previous studies may have been confounded by inter- and intraindividual variation. We believe this study provides an evidence indicating the impact of national policy on the pregnancy rate among young infertile women, as a form of quasi-experimental study.

As a retrospective study of a single IVF center, the findings have to be interpreted with caution. To apply our study design, we excluded all women aged outside of 34-35. Given the generally high pregnancy rate in this age group, our findings cannot be applied to older patients, who tend to have a less favorable prognosis. By analyzing young infertile women, however, we believe we have minimized the confounding effect of age and ovarian function in assessing the impact of additional ET. Second, we did not consider the quality of embryos in each cycle. Different quality/grades of transferred embryos in each cycle may have influenced the pregnancy outcome. However, as we used a mixedmodels approach, adjusting for intraindividual effects, we believe that we have reduced the potential confounding effects of heterogeneity of embryo grade. Lastly, we did not consider all the covariates such as insemination method and indication of IVF which can potentially confound the association between the number of transferred embryos and pregnancy rate. As we included only 34- and 35-year-old women, we believe these two age groups would be similar to each other in the basal characteristics.

\section{Conclusion}

We observed an increased probability of intrauterine pregnancy with additional ET only for frozen day 5-6 ET cycles. Therefore, we recommend transferring a smaller number of embryos per fresh ET cycle in 35-year-old women. Our study provides new insight to the old question of the number of embryos to transfer during IVF and 
should assist public policy for more optimal IVF pregnancy outcome.

\section{Acknowledgments}

We thank Sun-Wha Shim and Siha Jeong for their assistance throughout our study. This work was supported by the National Research Foundation of Korea grant (NRF2016R1D1A1B03933410 and 2018R1D1A1B07048821), which is funded by the Korean Government.

\section{Disclosure}

The authors report no conflicts of interest in this work.

\section{References}

1. Klitzman R. Deciding how many embryos to transfer: ongoing challenges and dilemmas. Reprod Biomed Soc Online. 2016;3:1-15. doi:10.1016/j.rbms.2016.07.001

2. Vega MG, Gleicher N, Darmon SK, et al. IVF outcomes in averageand poor-prognosis infertile women according to the number of embryos transferred. Reprod Biomed Online. 2016;33(3):370-375. doi:10.1016/j.rbmo.2016.06.009

3. Kissin DM, Kulkarni AD, Kushnir VA, Jamieson DJ; National ARTSSG. Number of embryos transferred after in vitro fertilization and good perinatal outcome. Obstet Gynecol. 2014;123(2 Pt 1):239-247. doi:10.1097/AOG.0000000000000106

4. Societies IFoF. IFFS Surveillance 2016; 2016:2473-3709.

5. Kutlu P, Atvar O, Vanlioglu OF, et al. Effect of the new legislation and single-embryo transfer policy in Turkey on assisted reproduction outcomes: preliminary results. Reprod Biomed Online. 2011;22 (2):208-214. doi:10.1016/j.rbmo.2010.10.007

6. Pandian Z, Marjoribanks J, Ozturk O, Serour G, Bhattacharya S. Number of embryos for transfer following in vitro fertilisation or intra-cytoplasmic sperm injection. Cochrane Database Syst Rev. 2013;7. doi:10.1002/14651858.CD003416.pub4
7. ESHRE. Regulation and Legislation in Assisted Reproduction; 2017.

8. Bissonnette F, Phillips SJ, Gunby J, et al. Working to eliminate multiple pregnancies: a success story in Québec. Reprod Biomed Online. 2011;23(4):500-504. doi:10.1016/j.rbmo.2011.05.020

9. Craig P, Katikireddi SV, Leyland A, Popham F. Natural experiments: an overview of methods, approaches, and contributions to public health intervention research. Annu Rev Public Health. 2017;38 (1):39-56. doi:10.1146/annurev-publhealth-031816-044327

10. Shen CT, Chen FM, Hsieh HM. Effect of a national population-based breast cancer screening policy on participation in mammography and stage at breast cancer diagnosis in Taiwan. Health Policy. 2020;124 (4):478-485. doi:10.1016/j.healthpol.2020.02.006

11. Niederkrotenthaler T, Sonneck G. Assessing the impact of media guidelines for reporting on suicides in Austria: interrupted time series analysis. Aust N Z J Psychiatry. 2007;41(5):419-428. doi:10.1080/ 00048670701266680

12. Pedersen NH, Koch S, Larsen KT, et al. Protocol for evaluating the impact of a national school policy on physical activity levels in Danish children and adolescents: the PHASAR study - a natural experiment. BMC Public Health. 2018;18(1):1245. doi:10.1186/s12889-018-6144-8

13. Division CP, MOWH. Maximum number of transferred embryos is reduced from 5 to 3. Announcement. 2015. Available from: http://www. mohw.go.kr/react/al/sal0301vw.jsp?PAR_MENU_ID=04\&MENU_ID= 0403\&CONT_SEQ=325206\&page=19. Accessed March 16, 2020.

14. Lee C Seoul sets advisory limit on IVF embryo transfer. The Korea Herald. 2015 Sept 2

15. Choe SA, Jun YB, Lee WS, Yoon TK, Kim SY. Association between ambient air pollution and pregnancy rate in women who underwent IVF. Hum Reprod. 2018;33(6):1071-1078. doi:10.1093/humrep/dey076

16. Baruffi RLR, Mauri AL, Petersen CG, et al. Single-embryo transfer reduces clinical pregnancy rates and live births in fresh IVF and Intracytoplasmic Sperm Injection (ICSI) cycles: a meta-analysis. Reprod Biol Endocrinol. 2009;7(1):36. doi:10.1186/1477-7827-7-36

17. Racca A, Drakopoulos P, Van Landuyt L, et al. Single and double embryo transfer provide similar live birth rates in frozen cycles. Gynecol Endocrinol. 2020;36(9):824-828.

\section{Publish your work in this journal}

The International Journal of Women's Health is an international, peerreviewed open-access journal publishing original research, reports, editorials, reviews and commentaries on all aspects of women's healthcare including gynecology, obstetrics, and breast cancer. The manuscript management system is completely online and includes a very quick and fair peer-review system, which is all easy to use. Visit http://www.dovepress.com/testimonials.php to read real quotes from published authors. 